\title{
XXVIII. Note on comparing capacities
}

\section{E.C. Rimington}

To cite this article: E.C. Rimington (1887) XXVIII. Note on comparing capacities, Philosophical Magazine Series 5, 24:148, 238-244, DOI: 10.1080/14786448708628088

To link to this article: http://dx.doi.org/10.1080/14786448708628088

$$
\text { 册 Published online: } 29 \text { Apr } 2009 .
$$

Submit your article to this journal

LII Article views: 2

Q View related articles $\asymp$ 
and we have an additional equation,

whence

$$
-\mathrm{X} \dot{x}_{0}=v / \mathrm{C}
$$

$$
v \mathrm{X}=c \mathrm{X}^{2} \dot{x}_{0}
$$

Thus the subsequent investigation is the same as that of the last section if we replace $\mathrm{L}$ by $\mathrm{CX}^{2}$, and the condition of zero-deflection is

$$
a \mathrm{M} b+d^{2}=\mathrm{CX}^{2} \mathrm{R} b^{2} \text {. }
$$

\section{Note on Comparing Capacities.}

By E. C. Rimington*.

TET two condensers, of capacities $K_{1}$ and $K_{2}$, be con1 nected to the arms $\mathrm{A}$ and $\mathrm{D}$ of a Wheatstone-bridge, as shown in woodcut; and let

$$
\mathrm{AC}=\mathrm{BD} \text {, }
$$

so that there is a balance for permanent currents.

Let $x+y, x, z$, and $u$ be the cyclic currents at some moment after the batterycircuit is broken, and when the condensers are dis. charging; and let $q_{1}$ be the charge on $K_{1}$ and $q_{\mathbf{s}}$ that on $K_{2}$ at this moment.

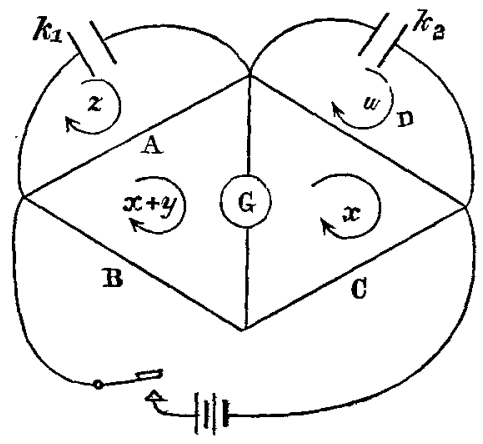
Then we have

also

$$
\begin{aligned}
& (\mathrm{A}+\mathrm{B}+\mathrm{G})(x+y)-\mathrm{G} x-\mathrm{A} z=0 \dagger \\
& (\mathrm{C}+\mathrm{D}+\mathrm{G}) x-\mathrm{G}(x+y)-\mathrm{D} w=0
\end{aligned}
$$

Therefore

$$
z=\frac{d q_{1}}{d t} \quad \text { and } \quad w=\frac{d q_{2}}{d t}
$$

$$
\begin{aligned}
(\mathrm{A}+\mathrm{B}) x+(\mathrm{A}+\mathrm{B}+\mathrm{G}) y & =\mathrm{A} \frac{d q_{1}}{d t}, \\
(\mathrm{C}+\mathrm{D}) x-\mathrm{G} y & =\mathrm{D} \frac{d q_{2}}{d t} .
\end{aligned}
$$

* Communicated by the Physical Society: read June 25, 1887.

+ The self-induction of the galvanometer need not be taken into account, as the current in it commences and ends at zero. The arms $A, B, C$, and $D$ are supposed to possess no self-induction. 
Hence

or

$$
y=\frac{\left|\begin{array}{rr}
\mathrm{A} \frac{d q_{1}}{d t}, & \mathrm{~A}+\mathrm{B} \\
\mathrm{D} \frac{d q_{2}}{d t}, & \mathrm{C}+\mathrm{D}
\end{array}\right|}{\left|\begin{array}{cc}
\mathrm{A}+\mathrm{B}+\mathrm{G}, & \mathrm{A}+\mathrm{B} \\
-\mathrm{G}, & \mathrm{C}+\mathrm{D}
\end{array}\right|}
$$

where

$$
\Delta y=\mathrm{A}(\mathrm{C}+\mathrm{D}) \frac{d q_{1}}{d t}-(\mathrm{A}+\mathrm{B}) \mathrm{D} \frac{d q_{2}}{d t}, .
$$

Integrating,

$$
\Delta=\mathrm{G}(\mathrm{A}+\mathrm{B})+(\mathrm{A}+\mathrm{B}+\mathrm{G})(\mathrm{C}+\mathrm{D}) .
$$

$$
\Delta \int_{0}^{\infty} y d t=\mathrm{A}(\mathrm{C}+\mathrm{D}) \int_{\mathrm{K}_{1} \mathbf{A} x_{0}}^{0} d q_{1}-(\mathrm{A}+\mathrm{B}) \mathrm{D} \int_{\mathrm{K}_{2} \mathrm{D} x_{0}}^{0} d q_{2},
$$

where $x_{0}$ is the permanent current through $\mathrm{A}$ and $\mathrm{D}$ when the battery is on.

But $\int_{0}^{\infty} y d t$ is the quantity which passes through the galvanometer, $=q$.

Therefore

$$
\Delta q=x_{0}\left\{\mathrm{~K}_{2} \mathrm{D}^{2}(\mathrm{~A}+\mathrm{B})-\mathrm{K}_{1} \mathrm{~A}^{2}(\mathrm{C}+\mathrm{D})\right\} .
$$

If $x_{1}$ is the permanent current in $\mathrm{B}$ and $\mathrm{C}$,

$$
x_{0}=x_{1} \frac{\mathrm{C}}{\mathrm{D}}
$$

and since $\mathrm{AC}=\mathrm{BD}$, we have

$$
q=x_{1} \mathrm{BD} \frac{\mathrm{K}_{2} \mathrm{C}-\mathrm{K}_{1} \mathrm{~B}}{\mathrm{G}(\mathrm{B}+\mathrm{C})+\mathrm{B}(\mathrm{C}+\mathrm{D})}, \ldots .
$$

which may be written

$$
q=x_{1} \frac{\mathrm{K}_{2} \mathrm{C}-\mathrm{K}_{1} \mathrm{~B}}{\frac{\mathrm{G}(\mathrm{B}+\mathrm{C})}{\mathrm{BD}}+\frac{\mathrm{C}}{\mathrm{D}}+1}
$$

If now $A$ and $D$ be made infinite, which will obviate the necessity of first adjusting for a permanent balance,

Also

$$
q=x_{1}\left(\mathrm{~K}_{2} \mathrm{C}-\mathrm{K}_{1} \mathrm{~B}\right) \text {. }
$$

$$
x_{1}=\frac{\mathrm{E}}{\rho+\mathrm{B}+\mathrm{C}},
$$


where $\rho$ is the internal resistance and $\mathrm{E}$ the E.M.F. of the testing battery.

If $q=0$,

$$
q=\frac{\mathrm{E}}{\rho+\mathrm{B}+\mathrm{C}}\left(\mathrm{K}_{2} \mathrm{C}-\mathrm{K}_{1} \mathrm{~B}\right)
$$

$$
\frac{\mathrm{K}_{1}}{\mathrm{~K}_{2}}=\frac{\mathrm{C}}{\mathrm{B}}
$$

If a key be also put in the galvanometer-circuit, and the battery-key be first depressed and then, after a certain interval of time, the galvanometer-key, we have Gott's method of comparing capacities. This allows the condenser longer time to charge; and if the galvanometer when its key is depressed shows no throw, it indicates that the potentials $v_{1}$ and $v_{2}$ are equal, and that therefore

as before.

$$
\frac{\mathrm{K}_{1}}{\mathrm{~K}_{2}}=\frac{\mathrm{C}}{\mathrm{B}}
$$

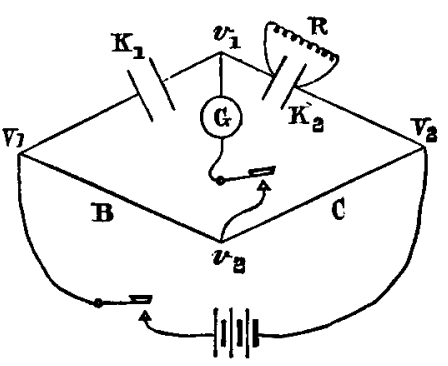

If, however, either of the condensers has an appreciable leakage, the result will be false by this method.

Suppose the condenser $K_{2}$ has an insulation-resistance $R$, that of $K_{1}$ being infinite. On depressing the battery-key, as the condensers are in series, they take initial charges each equal to $\mathrm{Q}$.

$$
\mathrm{Q}=\left(\nabla_{1}-v_{1}\right) \mathrm{K}_{1}=\left(v_{1}-\mathrm{V}_{2}\right) \mathrm{K}_{2}=\left(\mathrm{V}_{1}-\mathrm{V}_{2}\right) \frac{\mathrm{K}_{1} \mathrm{~K}_{2}}{\mathrm{~K}_{1}+\mathrm{K}_{2}} \text {. }
$$

Let an interval of time $t$ elapse before the galvanometer-key is depressed, and at the end of it let $Q_{1}$ and $Q_{2}$ be the charges on $\mathrm{K}_{1}$ and $\mathrm{K}_{2}$ respectively, and $v$ the value of $v_{1}$. Then

$$
\begin{aligned}
& \mathrm{Q}_{2}=\mathrm{Q} e^{-\frac{t}{\mathrm{~K}_{2} \mathrm{R}}}, \text { where } e=2 \cdot 718, \\
& \mathrm{Q}_{2}=\left(\mathrm{V}_{1}-\mathrm{V}_{2}\right) \frac{\mathrm{K}_{1} \mathrm{~K}_{2}}{\mathrm{~K}_{1}+\mathrm{K}_{2}} e^{-\frac{t}{\mathrm{~K}_{2} \mathrm{R}}}
\end{aligned}
$$

and

$$
\begin{aligned}
& \mathrm{Q}_{1}=\left(\mathrm{V}_{1}-v\right) \mathrm{K}_{1}=\left\{\left(\mathrm{V}_{1}-\mathrm{V}_{2}\right)-\left(v-\mathrm{V}_{2}\right) \mathrm{K}_{1}\right. \\
&=\left\{\left(\mathrm{V}_{1}-\mathrm{V}_{2}\right)-\frac{\mathrm{Q}_{2}}{\mathrm{~K}_{2}}\right\} \mathrm{K}_{1}, \\
& \mathrm{Q}_{2}=\left(\mathrm{V}_{1}-\mathrm{V}_{2}\right)\left(1-\frac{\mathrm{K}_{1}}{\mathrm{~K}_{1}+\mathrm{K}_{2}} e^{-\frac{t}{\mathrm{~K}_{2} \mathrm{R}}}\right) \mathrm{K}_{1} .
\end{aligned}
$$


Now

and

$$
\left(v-\mathrm{V}_{2}\right)=\frac{\mathrm{Q}_{2}}{\mathrm{~K}_{2}}=\left(\mathrm{V}_{1}-\mathrm{V}_{2}\right) \frac{\mathrm{K}_{1}}{\mathrm{~K}_{1}+\overline{\mathrm{K}}_{2}} e^{-\frac{t}{\mathrm{~K}_{2} \mathrm{R}}}
$$

Hence

$$
\left(V_{1}-v\right)=\frac{Q_{1}}{K_{1}}=\left(V_{1}-V_{2}\right)\left(1-\frac{K_{1}}{K_{1}+\bar{K}_{2}} e^{-\frac{t}{K_{2} \bar{R}}}\right) \text {. }
$$

Also

$$
\frac{v-\mathrm{V}_{2}}{\overline{\nabla_{1}-v}}=\frac{\mathrm{K}_{1} e^{-\frac{t}{\mathrm{~K}_{2} \mathrm{R}}}}{\mathrm{K}_{1}\left(1-e^{-\frac{t}{\mathrm{~K}_{2} \mathrm{R}}}\right)+\mathrm{K}_{2}}
$$

$$
\frac{v_{2}-\mathrm{V}_{2}}{\overline{\mathrm{V}}_{1}-v_{2}}=\frac{\mathrm{C}}{\mathrm{B}}
$$

If, therefore, $v=v_{2}$, or there appears to be a balance,

$$
\overline{\mathrm{B}}=\frac{\mathrm{K}_{1} e^{-\frac{t}{\mathrm{~K}_{2} \mathrm{R}}}}{\mathrm{K}_{1}\left(1-e^{-\frac{t}{\mathrm{~K}_{2} \mathrm{R}}}\right)+\mathrm{K}_{2}}
$$

or

$$
\mathrm{K}_{1}=\frac{\mathrm{K}_{2} \mathrm{C}}{(\mathrm{B}+\mathrm{C}) e^{-\frac{t}{\mathrm{~K}_{2} \mathrm{R}}}-\mathrm{C}} \text {. }
$$

So that, unless $t$ be made very small compared to $K_{2} R$, we cannot compare $\mathrm{K}_{1}$ and $\mathrm{K}_{2}$ unless we know $\mathrm{R}$ and $t$. In the case of no leakage the conditions will be the same as if there were no key in the galvanometer circuit.

To find the best conditions for the test:-Taking equation (2), and substituting for $x_{1}$ its value

$$
\frac{\mathrm{E}}{\rho+\frac{(\mathrm{A}+\mathrm{D})(\mathrm{B}+\mathrm{C})}{\mathrm{A}+\mathrm{D}+\mathrm{B}+\mathrm{C}}} \times \frac{\mathrm{D}}{\mathrm{C}+\overline{\mathrm{D}}},
$$

which equals $\frac{\mathrm{ED}}{\rho(\mathrm{C}+\mathrm{D})+\mathrm{D}(\mathrm{B}+\mathrm{C})}$, since $\mathrm{AC}=\mathrm{BD}$, we obtain

$$
q=\frac{\mathrm{EBD}^{2}\left(\mathrm{~K}_{2} \mathrm{C}-\mathrm{K}_{1} \mathrm{~B}\right)}{\{\rho(\mathrm{C}+\mathrm{D})+\mathrm{D}(\mathrm{B}+\mathrm{C})\}\{\mathrm{G}(\mathrm{B}+\mathrm{C})+\mathrm{B}(\mathrm{C}+\mathrm{D})\}} .
$$

If $q=0, \mathrm{~K}_{2} \mathrm{C}=\mathrm{K}_{1} \mathrm{~B}$.

Suppose a fractional error $p$ to be made in the value of $\mathrm{C}$ Phil. Mag. S. 5. Vol. 24. No. 148. Sept. 1887. $\mathrm{R}$ 
Then

$$
\begin{aligned}
q & =\frac{\mathrm{EBD}^{2} p \mathrm{~K}_{2} \mathrm{C}}{\{\rho(\mathrm{C}+\mathrm{D})+\mathrm{D}(\mathrm{B}+\mathrm{C})\}\{\mathrm{G}(\mathrm{B}+\mathrm{C})+\mathrm{B}(\mathrm{C}+\overline{\mathrm{D}})\}} \\
& =\frac{\mathrm{E} p \mathrm{~K}_{2}}{\left(\frac{\rho}{\mathrm{D}}+\frac{\rho}{\mathrm{C}}+\frac{\mathrm{B}+\mathrm{C}}{\mathrm{C}}\right)\left(\frac{\mathrm{G}}{\mathrm{D}}+\frac{\mathrm{GC}}{\mathrm{BD}}+\frac{\mathrm{C}+\mathrm{D}}{\mathrm{D}}\right)} .
\end{aligned}
$$

Now the throw is proportional to $q \sqrt{ } \mathrm{G}$ for a galvanometer whose coil-volume is constant. Therefore the throw is proportional to

$$
\frac{\mathrm{E} p \mathrm{~K}_{2}}{\left(\frac{\rho}{\mathrm{D}}+\frac{\rho}{\mathrm{C}}+\frac{\mathrm{B}+\mathrm{C}}{\mathrm{C}}\right)\left(\frac{\sqrt{ } \mathrm{G}}{\mathrm{D}}+\frac{\mathrm{C} \sqrt{ } \mathrm{G}}{\mathrm{BD}}+\frac{\mathrm{C}+\mathrm{D}}{\mathrm{D} \sqrt{\mathrm{G}}}\right)},
$$

and this is to be a maximum; which it obviously is when

$$
\checkmark \mathrm{G} \cdot \frac{\mathrm{B}+\mathrm{C}}{\mathrm{BD}}=\frac{\mathrm{C}+\mathrm{D}}{\mathrm{D}}-\frac{1}{\sqrt{ } \mathrm{G}}, \quad \text { or } \quad \mathrm{G}=\frac{\mathrm{B}(\mathrm{C}+\mathrm{D})}{\mathrm{B}+\mathrm{C}} \text {. }
$$

If $A$ and $D$ are infinite, the higher the resistance of the galvanometer the better. Also, in this case,

$$
q=\frac{\mathrm{E} p \mathrm{~K}_{2}}{\frac{\rho}{\mathrm{C}}+\frac{\mathrm{B}}{\overline{\mathrm{C}}}+1}=\frac{\mathrm{E} p \mathrm{~K}_{2}}{\frac{\rho}{\mathrm{C}}+\frac{\mathrm{K}_{2}}{\mathrm{~K}_{1}}+1} .
$$

Here $q$ is largest when $\mathrm{C}$ is largest.

To find the conditions under which a telephone may replace the galvanometer :-

In equation (1) we have

$$
\Delta y=\mathrm{A}(\mathrm{C}+\mathrm{D}) \frac{d q_{1}}{d t}-(\mathrm{A}+\mathrm{B}) \mathrm{D} \frac{d q_{2}}{d t} .
$$

Now, in order to employ a telephone, $y$ must always be zero. Therefore

But

$$
\frac{\mathrm{A}}{\mathrm{A}+\mathrm{B}} \frac{d q_{1}}{d t}=\frac{\mathrm{D}}{\mathrm{C}+\mathrm{D}} \frac{d q_{2}}{d t}
$$

Hence

$$
\frac{A}{A+B}=\frac{D}{C+D}, \text { since } A C=B D \text {. }
$$

$$
\frac{d q_{1}}{d t}=\frac{d q_{2}}{d t}
$$

Now, since there is no current in the galvanometer, its resistance may be anything: let it be zero. Then $K_{1}$ discharges through $\frac{A B}{A+B}$, and $K_{2}$ through $\frac{C D}{A+D}$. 
Consequently

and

$$
q_{1}=x_{0} \mathrm{~K}_{1} \mathrm{~A} e^{-\frac{t}{\mathrm{~K}_{1} \frac{\mathrm{AB}}{\mathrm{A}+\overline{\mathrm{B}}}}}
$$

Hence

$$
q_{2}=x_{0} \mathrm{~K}_{2} \mathrm{D} e^{\frac{t}{\mathrm{~K}_{2} \frac{\mathrm{CD}}{\mathrm{C}+\mathrm{D}}}}
$$

$$
\begin{aligned}
\frac{d q_{1}}{d t} & =-x_{0} \mathrm{~K}_{1} \mathrm{~A} \cdot \frac{\mathrm{A}+\mathrm{B}}{\mathrm{K}_{1} \mathrm{AB}} e^{-\frac{t}{\mathrm{~K}_{1} \frac{\mathrm{A} B}{\mathrm{~A}+\mathrm{B}}}} \\
& =-x_{0} \frac{\mathrm{A}+\mathrm{B}}{\mathrm{B}} e^{-\frac{t}{\mathrm{~K}_{1} \frac{\mathrm{AB}}{\mathrm{A}+\mathrm{B}}}}
\end{aligned}
$$

and

Therefore

$$
\frac{d q_{2}}{d t}=-x_{0} \frac{\mathrm{C}+\mathrm{D}}{\mathrm{C}} e^{-\frac{t}{\mathrm{~K}_{2} \frac{\mathrm{CD}}{\mathrm{C}+\mathrm{D}}}}
$$

and

$$
\frac{\mathrm{A}+\mathrm{B}}{\mathrm{B}} e^{-\frac{t}{\mathrm{~K}_{1} \frac{\mathrm{AB}}{\mathrm{A}+\mathrm{B}}}}=\frac{\mathrm{C}+\mathrm{D}}{\mathrm{C}} e^{-\frac{t}{\mathrm{~K}_{2} \frac{\mathrm{CD}}{\mathrm{C}+\mathrm{D}}}}
$$

Therefore

$$
\frac{A+B}{B}=\frac{C+D}{D}
$$

and, since $\mathrm{AC}=\mathrm{BD}$,

$$
K_{1} \frac{A B}{A+B}=K_{2} \frac{C D}{C+D}
$$

$$
\begin{gathered}
\mathrm{K}_{1} \mathrm{~B}=\mathrm{K}_{2} \mathrm{C}, \\
\frac{\mathrm{K}_{1}}{\mathrm{~K}_{2}}=\frac{\mathrm{C}}{\mathrm{B}} .
\end{gathered}
$$

If there is self-induction in the four conductors, let $L_{1}$ be the coefficient of self-induction of $\mathrm{A}, \mathrm{L}_{2}$ of $\mathrm{D}, \mathrm{L}_{3}$ of $\mathrm{B}$, and $\mathrm{L}_{4}$ of $\mathrm{C}$. Then

$$
\begin{aligned}
(\mathrm{A}+\mathrm{B}+\mathrm{G}) y+(\mathrm{A}+\mathrm{B}) x+\mathrm{I}_{1} \frac{d(x+y)}{d t}+\mathrm{L}_{3} \frac{d(x+y)}{d t}-\mathrm{A} \frac{d q_{1}}{d t} & =0 . \\
-\mathrm{G} y+(\mathrm{C}+\mathrm{D}) x+\mathrm{L}_{2} \frac{d x}{d t}+\mathrm{L}_{4} \frac{d x}{d t}-\mathrm{D} \frac{d q_{2}}{d t} & =0 .
\end{aligned}
$$

Solving for $y$, we have

$$
\begin{gathered}
\Delta y=(\mathrm{C}+\mathrm{D})\left\{\mathrm{A} \frac{d q_{1}}{d t}-\mathrm{L}_{1} \frac{d(x+y)}{d t}-\mathrm{L}_{3} \frac{d(x+y)}{d t}\right\} \\
-(\mathrm{A}+\mathrm{B})\left\{\mathrm{D} \frac{d q_{2}}{d t}-\mathrm{L}_{2} \frac{d x}{d t}-\mathrm{L}_{4} \frac{d x}{d t}\right\} . \\
\mathrm{R} 2
\end{gathered}
$$


Integrating,

$$
\begin{aligned}
& \Delta q=(\mathrm{C}+\mathrm{D})\left\{\mathrm{A} \int_{\mathbb{K}_{1} \mathrm{~A} x_{0}}^{0} d q_{1}-\mathrm{L}_{1} \int_{x_{0}}^{0} d(x+y)-\mathrm{L}_{3} \int_{-\overline{\mathrm{B}} x_{0}}^{0} d(x+y)\right\} \\
& -(\mathrm{A}+\mathrm{B})\left\{\mathrm{D} \int_{\mathrm{K}_{2} \mathrm{D} x_{0}}^{0} d q_{2}-\mathrm{I}_{2} \int_{x_{0}}^{0} d x-\mathrm{L}_{4} \int_{-\overline{\mathbf{A}} x_{0}}^{0} d x\right\} \\
& =x_{0} \frac{\mathrm{C}+\mathrm{D}}{\mathrm{C}^{2}}\left(\mathrm{BCD}^{2} \mathrm{~K}_{2}-\mathrm{B}^{2} \mathrm{D}^{2} \mathrm{~K}_{1}+\mathrm{L}_{1} \mathrm{C}^{2}-\mathrm{L}_{3} \mathrm{CD}-\mathrm{L}_{2} \mathrm{BC}+\mathrm{L}_{4} \mathrm{BD}\right),
\end{aligned}
$$

Also

$$
x_{0}=x_{1} \frac{\mathrm{C}}{\mathrm{D}}
$$

where $x_{1}$ is the permanent current in the branches $B$ and $C$.

$$
\therefore \Delta q=x_{1} \cdot \frac{\mathrm{C}+\mathrm{D}}{\mathrm{CD}}\left(\mathrm{BCD}^{2} \mathrm{~K}_{2}-\mathrm{B}^{2} \mathrm{D}^{2} \mathrm{~K}_{1}+\mathrm{L}_{1} \mathrm{C}^{2}-\mathrm{L}_{3} \mathrm{CD}\right.
$$

If $q=0$,

$$
\left.-\mathrm{L}_{2} \mathrm{BC}+\mathrm{L}_{4} \mathrm{BD}\right) \text {. }
$$

$$
\mathrm{BD}^{2}\left(\mathrm{~K}_{2} \mathrm{C}-\mathrm{K}_{1} \mathrm{~B}\right)+\mathrm{C}\left(\mathrm{L}_{1} \mathrm{C}-\mathrm{L}_{3} \mathrm{D}\right)+\mathrm{B}\left(\mathrm{L}_{4} \mathrm{D}-\mathrm{L}_{2} \mathrm{C}\right)=0 .
$$

Hence

or

$$
\mathrm{K}_{2} \mathrm{C}=\mathrm{K}_{1} \mathrm{~B}, \quad \mathrm{~L}_{1} \mathrm{C}^{\psi}=\mathrm{L}_{3} \mathrm{D}, \quad \mathrm{L}_{4} \mathrm{D}=\mathrm{L}_{2} \mathrm{C} \text {; }
$$

Again, since

$$
\frac{\mathrm{K}_{1}}{\mathrm{~K}_{2}}=\frac{\mathrm{C}}{\mathrm{B}} \quad \text { and } \quad \frac{\mathrm{L}_{1}}{\mathrm{~L}_{3}}=\frac{\mathrm{L}_{2}}{\mathrm{~L}_{4}}=\frac{\mathrm{D}}{\mathrm{C}} .
$$

$$
\begin{gathered}
\Delta=\frac{\mathrm{C}+\mathrm{D}}{\mathrm{C}}\{\mathrm{B}(\mathrm{C}+\mathrm{D})+\mathrm{G}(\mathrm{B}+\mathrm{C})\}, \\
q=x_{1} \frac{\mathrm{BCDK}_{2}-\mathrm{B}^{2} \mathrm{DK}_{1}+\mathrm{L}_{1} \frac{\mathrm{C}^{2}}{\mathrm{D}}-\mathrm{L}_{2} \frac{\mathrm{BC}}{\mathrm{D}}-\mathrm{L}_{3} \mathrm{C}+\mathrm{L}_{4} \mathrm{~B}}{\mathrm{~B}(\mathrm{C}+\mathrm{D})+\mathrm{G}(\mathrm{B}+\mathrm{C})} \\
=x_{1} \frac{\mathrm{K}_{2} \mathrm{BC}-\mathrm{K}_{1} \mathrm{~B}^{2}+\mathrm{L}_{1} \frac{\mathrm{C}^{2}}{\mathrm{D}^{2}}-\mathrm{L}_{2} \frac{\mathrm{BC}}{\mathrm{D}^{2}}-\mathrm{L}_{3} \frac{\mathrm{C}}{\mathrm{D}}+\mathrm{L}_{4} \frac{\mathrm{B}}{\mathrm{D}}}{\frac{\mathrm{BC}}{\mathrm{D}}+\mathrm{B}+\frac{\mathrm{G}(\mathrm{B}+\mathrm{C})}{\mathrm{D}}} .
\end{gathered}
$$

If $\mathrm{A}$ and $\mathrm{D}$ are now made infinite,

$$
q=x_{1}\left(\mathrm{~K}_{2} \mathrm{C}-\mathrm{K}_{1} \mathrm{~B}\right) ;
$$

the same result that we obtain if there is no self-induction in the branches $\mathrm{B}$ and $\mathrm{C}$. 